\title{
A case of Stewart-Treves syndrome occurring in the abdominal wall successfully treated with eribulin: A case report
}

\author{
YOSHINORI IMURA $^{1}$, SHIGENORI NAGATA ${ }^{2}$, TORU WAKAMATSU ${ }^{1}$, TAKAAKI TANAKA ${ }^{1}$, \\ HIRONARI TAMIYA $^{1}$, NORIFUMI NAKA ${ }^{1}$ and SATOSHI TAKENAKA ${ }^{1}$ \\ ${ }^{1}$ Musculoskeletal Oncology Service and ${ }^{2}$ Department of Diagnostic Pathology and \\ Cytology, Osaka International Cancer Institute, Osaka 541-8567, Japan
}

Received March 27, 2020; Accepted July 15, 2020

DOI: $10.3892 / \mathrm{mco} .2020 .2119$

\begin{abstract}
Angiosarcoma (AS) is a rare and aggressive tumor with high rates of local recurrence and distant metastasis. Stewart-Treves syndrome (STS) is defined as AS arising in the setting of chronic lymphedema, and is extremely uncommon in the lower abdominal wall. Eribulin mesylate (eribulin) is a non-taxane microtubule inhibitor that has been approved in Japan for treating soft tissue sarcoma. The current study reports the case of a 76 year-old woman with STS in the lower abdominal wall who exhibited an excellent response to eribulin. Having undergone surgery and postoperative radiation therapy (RT) for cervical cancer 12 years earlier, the patient presented with a mass in her left lower abdominal wall, where chronic lymphedema had developed. Contrast-enhanced computed tomography revealed multiple enhancing nodules in the left lower abdominal wall and edema of the subcutaneous tissues in the whole lower abdomen. A histologic analysis of the specimens revealed AS, and she was diagnosed as STS. A total of 3 cycles of combination chemotherapy with gemcitabine and docetaxel were administered, but the patient discontinued treatment owing to severe adverse events. RT was performed for the tumor, but multiple reddish nodules appeared in the whole lower abdominal wall 3 months later. At this point, eribulin administration was offered. After 4 cycles of treatment, there was a clear reduction in the size of the nodules. All lesions were stable, no new lesions had developed, and the side effects of treatment were minor over the course of 1 year. The results reveal that eribulin may serve as a potential therapeutic option for the treatment of STS.
\end{abstract}

Correspondence to: $\mathrm{Dr}$ Yoshinori Imura, Musculoskeletal Oncology Service, Osaka International Cancer Institute, 3-1-69 Otemae, Chuo-ku, Osaka 541-8567, Japan

E-mail: y.imura@hotmail.co.jp

Key words: angiosarcoma, Stewart-Treves syndrome, chronic lymphedema, eribulin, cervical cancer

\section{Introduction}

Angiosarcoma (AS), a highly malignant and rare tumor of vascular or lymphatic endothelial cell origin with a poor prognosis (1), arises in the scalp and face in the elderly, in association with chronic lymphedema, and in irradiated areas $(1,2)$. AS associated with chronic lymphedema is known as Stewart-Treves syndrome (STS) (2). STS is primarily described in patients with lymphedema of an upper extremity occurring after breast cancer surgery including radical axially lymph node dissection and subsequent radiation therapy (RT). STS accounts for approximately $5 \%$ of AS (3), and is rarely described in the presence of chronic lymphedema of the lower abdominal wall.

Eribulin mesylate (eribulin) is a structurally modified analog of halichondrin $\mathrm{B}$, originally isolated from the marine sponge Halichondria okadai. Its mode of action is distinct from other tubulin inhibitors and involves binding to specific sites on the growing positive ends of microtubules to inhibit their growth (4-6). Eribulin was approved in Japan for the treatment of patients with soft tissue sarcoma in 2016. The efficacy of eribulin for AS has not been evaluated sufficiently till date.

Herein, we report a rare case of STS in the lower abdominal wall successfully treated with eribulin.

\section{Case report}

The patient, a 76-year-old woman, underwent a radical hysterectomy with bilateral salpingo-ophorectomy and pelvic lymphadenectomy for cervical cancer 12 years earlier. Postoperative RT of 50.4 Gy in 28 fractions was administered. After treatment, lymphedema progressed in her lower abdominal wall and bilateral legs and she was treated with antibiotics for cellulitis repeatedly. Then, she noticed a mass on her left lower abdominal wall, where chronic lymphedema had developed. The mass grew rapidly for 3 months and she was referred to our outpatient clinic.

Magnetic resonance imaging (MRI) demonstrated that the mass was heterogenous isointense on T1-weighted image and heterogenous hyperintense on T2 weighted image (Fig. 1A). Diffuse thickening of cutis and subcutis as well as edema of subcutaneous tissues could be seen in the abdomen, groin, buttocks, and legs. Multiple enhancing nodules in the left 

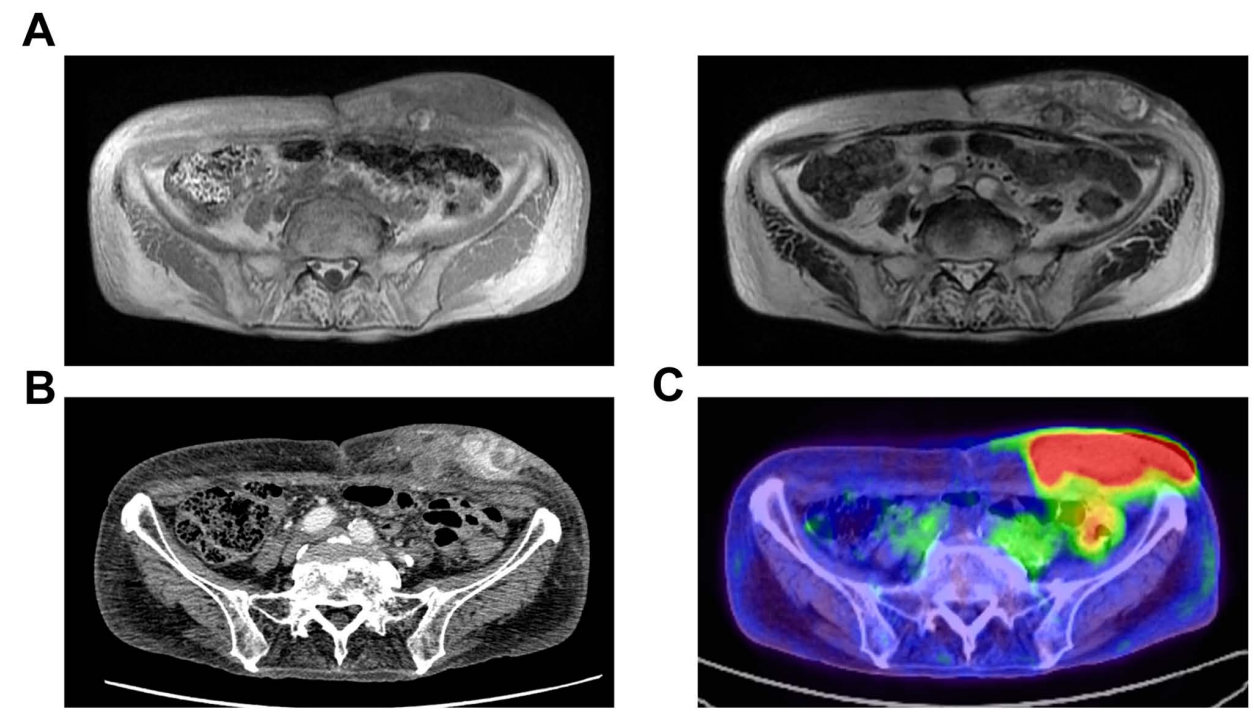

Figure 1. (A) MRI of the abdomen. Axial T1-weighted image showed that the mass was heterogenous iso-intensity signal and axial T2-weighted image showed that the mass was heterogenous high intensity signal. Diffuse thickening of the cutis and subcutis as well as edema of subcutaneous tissues were detected. Left, axial view on T1-weighted image; right, axial view on T2-weighted image. (B) Contrast-enhanced CT of the abdomen. Enhancing multiple nodules in the left lower abdominal wall and edema of the subcutaneous tissues were detected. (C) Fluorodeoxyglucose positron emission tomography/CT scan. Increased uptake corresponding to the mass in the left lower abdominal wall was seen (maximum standardized uptake values=22.0). MRI, magnetic resonance imaging; CT, computed tomography.
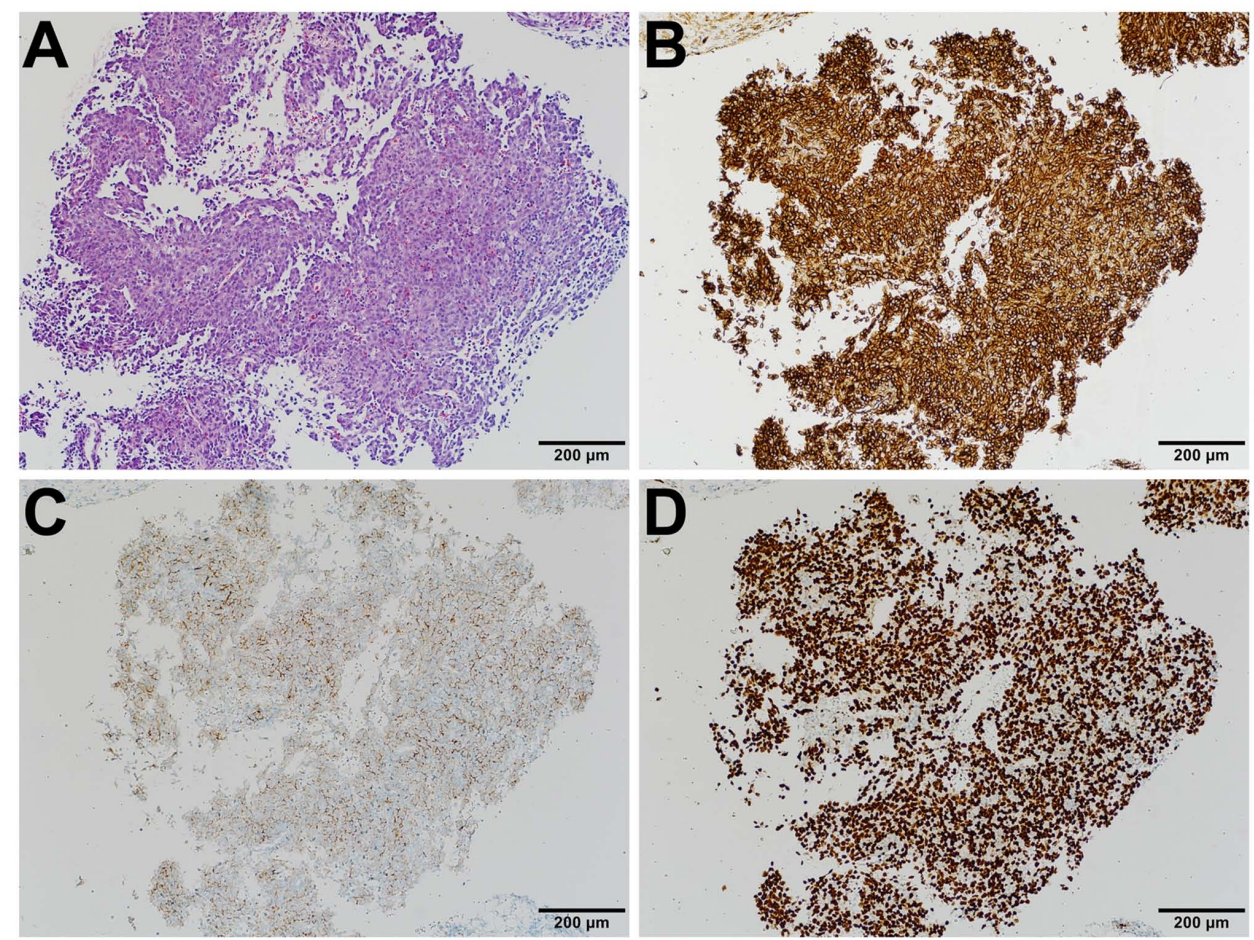

Figure 2. Histopathology staining of the needle biopsy specimens. (A) Hematoxylin and eosin staining showed that the immature vascular structures are formed with atypical epithelioid and spindle cells; magnification, x100. (B) Immunohistological staining for CD31, (C) D2-40 and (D) ERG were positive; magnification, $\mathrm{x} 100 . \mathrm{CD} 31$, cluster of differentiation.

lower abdominal wall and edema of subcutaneous tissues were revealed by contrast-enhanced computed tomography (CT) scan of the abdomen (Fig. 1B). On fluorodeoxyglucose positron emission tomography (FDG-PET)/CT scan, hypermetabolism (maximum standardized uptake values $=22.0$ ) was observed in the mass of the left lower abdominal wall (Fig. 1C), but distant metastasis was not detected. Immunohistologic examination of needle biopsy specimens of AS tested positive for CD31,
D2-40, and ERG (Fig. 2A-D). Based on the morphology, immunoprofile, and clinical presentation, the patient was diagnosed with STS.

We judged the tumor unresectable and the combination chemotherapy with gemcitabine $\left(720 \mathrm{mg} / \mathrm{m}^{2}\right)$ and docetaxel $\left(80 \mathrm{mg} / \mathrm{m}^{2}\right.$ ) was started. She demonstrated stable disease (SD) for 3 months. However, grade 4 neutropenia and anemia were observed. In addition, severe side effects such as fatigue, 
A
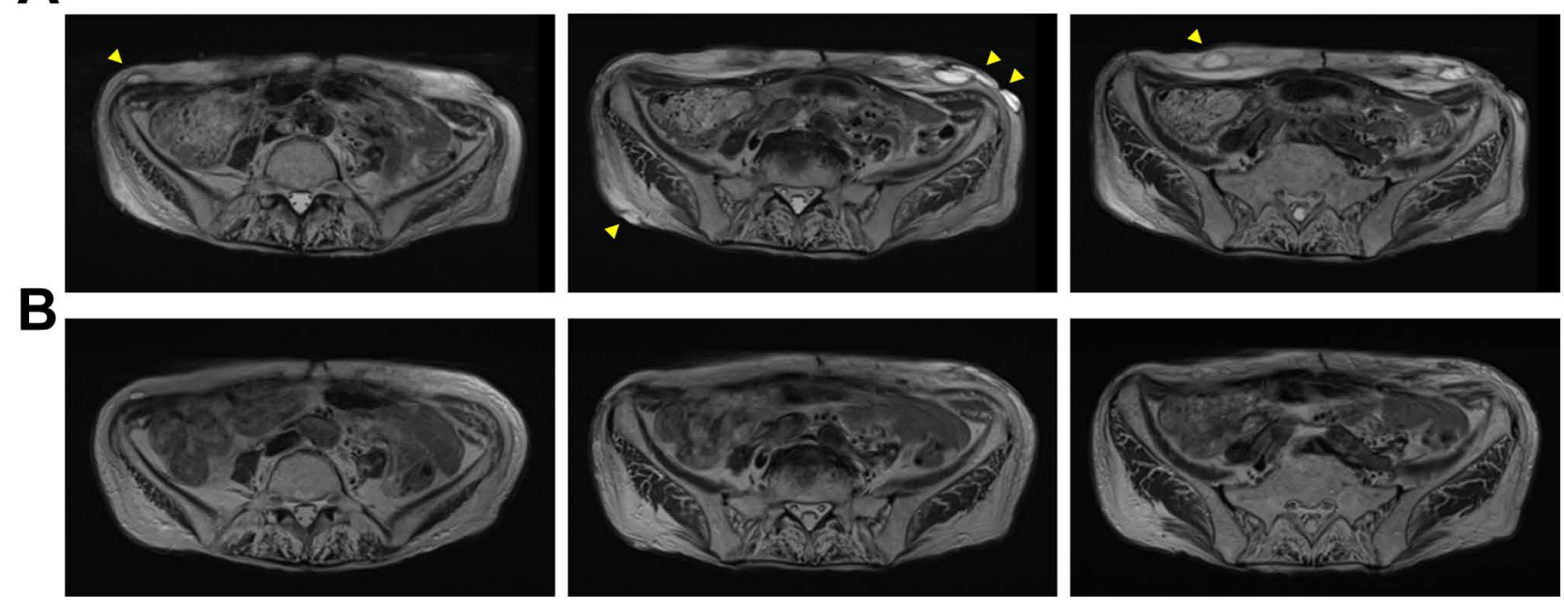

Figure 3. (A) MRI of the abdomen before eribulin administration. Multiple nodules (yellow arrows) were observed in the whole abdominal wall. (B) MRI of the abdomen 1 year after eribulin administration. Most nodules decreased in size. MRI, magnetic resonance imaging.

diarrhea, anorexia, vomiting, and weight loss occurred, and thus, the patient discontinued treatment after 3 cycles. Then, RT of 70 Gy in 35 fractions was carried out for the left lower abdominal wall tumor. The size of the mass decreased once but multiple reddish nodules, some of which were diagnosed as AS by excisional biopsies, developed in the whole abdominal wall 3 months after RT (Fig. 3A). At this point, treatment with eribulin was initiated at a dose of $1.4 \mathrm{mg} / \mathrm{m}^{2}$. However, grade 3 and 4 neutropenia occurred on day 8 and 15, respectively. In addition, anorexia and diarrhea continued for 1 week after eribulin administration. Then, the dose was gradually reduced. Eribulin was dosed at $0.8 \mathrm{mg} / \mathrm{m}^{2}$ and was administered on day 1 of a 21-day cycle for 10 months. There was shrinkage of nodules in the lower abdominal wall after 4 courses of eribulin treatment, and no new lesions have developed. Treatment with eribulin is effective with tumor partial response (PR). MRI demonstrated that these effects lasted for 1 year without severe side effects (Fig. 3B), and she still continues to receive eribulin treatment.

\section{Discussion}

AS is a rare malignant tumor with a predilection for skin in the head and neck region, though it can appear anywhere on the body and has often been described as looking like a skin infection, a bruise, or a lesion (1). Its association with chronic lymphedema is well known $(1,2)$. STS was first described when Fred Stewart and Norman Treves reported a case series describing 6 patients with AS in the setting of chronic lymphedema that developed post radical mastectomy (2). Although it is widely accepted that lymphedema may induce AS, the mechanism remains unknown. Local immunodeficiency resulted from long-standing chronic lymphedema may be involved in oncogenesis of STS, and the development of AS has been associated with chronic lymphedema of any origin. STS typically occurs as a complication of long-lasting lymphedema of the arm after axillary lymph node dissection following a radical mastectomy and/or RT for breast cancer. STS can also appear in lower limbs with chronic lymphedema. However, STS has rarely been described in the presence of chronic lymphedema of the lower abdominal wall and postoperative cervical cancer. For women with cervical cancers, surgery that removes lymph nodes and/or RT to the pelvis may damage lymph nodes in that area and lymphedema often develops in the legs, abdomen, or groin area. Severe cellulitis infection may damage tissue around the lymph nodes or vessels and increase the risk of lymphedema. In this case, cellulitis occurred repeatedly in the lower abdomen, probably leading to lymphedema.

To date, more than 400 cases of STS have been reported in the English literature. Collectively, STS has very poor prognosis and is prone to local recurrence and distant metastasis $(1,7,8)$. Because of the occurrence of metastatic spread and poor prognosis, proper diagnosis of AS is important. Due to the rarity of STS, there is no standardized therapy for this disease. Radical surgery by local resection with a negative margin is the only curable treatment available and complete tumor resection predicts improved prognosis $(9,10)$. Therefore, once the biopsy specimen has been diagnosed as STS, extensive resection, early amputation, or dissection of the joint and diseased limb are highly recommended, which may increase the survival rate of patients. However, in our case, we judged the tumor unresectable with a safety margin. The median survival is 2.5 years after diagnosis, with most patients dying from metastatic disease within 2 years $(1,3,7,8)$. The long-term survivorship is rare, and survival time of untreated patients is only 5-8 months post diagnosis.

AS is a highly aggressive and exceedingly rare soft tissue sarcoma. Despite a strong need for systemic therapies, the rarity of AS represents a major limitation to randomized trials and therefore very few prospective clinical studies focusing on the medical treatment are available. Standard chemotherapy for soft tissue sarcoma is based on anthracyclines as the first line treatment (11). Beside anthracyclines, taxanes can be active, both as single agents and in combination with gemcitabine in AS (12-19). Taxanes are the current first line treatment for patients with advanced AS who are considered difficult to treat with anthracyclines owing to advanced age or comorbidity. Anthracyclines can be difficult to administer in this patient due to high age and cardiac dysfunction. Tolerability of gemcitabine plus docetaxel is fair, with less cardiac toxicity 
compared with anthracyclines. Therefore, we administered combination therapy of gemcitabine and docetaxel in this patient.

Eribulin, a nontaxane microtubule-targeting agent, was originally approved for the treatment of breast cancer by the United States Food and Drug Administration in 2010. A phase III study comparing eribulin with dacarbazine in patients with advanced liposarcoma or leiomyosarcoma showed significant improvement in overall survival (OS) for the eribulin arm, with a manageable toxicity profile $(20,21)$. In the liposarcoma subgroup, OS was significantly improved with eribulin vs dacarbazine (15.6 vs. 8.4 months) and progression free survival was also improved with eribulin vs. dacarbazine (2.9 vs. 1.7 months) $(20,21)$. Based on the results of this phase III study, in 2016 eribulin was approved for the treatment of patients with unresectable or metastatic liposarcoma who have received a prior anthracycline-containing regimen in both the United States and Europe. On the other hand, exploratory analysis from a Japanese phase II study showed some efficacy of eribulin in patients with several subtypes of rare soft tissue sarcoma including synovial sarcoma, endometrial stromal sarcoma, solitary fibrous tumor, and fibrosarcoma (22). As a result, eribulin was approved for the treatment of all soft tissue sarcomas in Japan. However, little data exists regarding eribulin efficacy in rare histologic subtypes of soft tissue sarcomas such as AS.

Recently, 2 case reports from Japan described patients with AS whose disease was controlled successfully with eribulin mesylate $(23,24)$. Kobayashi et al demonstrated interim results of a post-marketing surveillance study of eribulin in soft tissue sarcomas including rare subtypes such as AS (25). In that study, of 12 AS patients, PR was achieved in 1 patient (8.3\%) and SD was achieved in 2 patients (16.7\%). However, treatment duration of eribulin in those 3 patients was less than 9 months. Fujisawa et al showed the promising efficacy of eribulin for treating patients with advanced cutaneous AS who previously received taxanes (26). The best overall response rate was $20 \%$, and the median overall survival and progression-free survival were 8.6 and 3.0 months, respectively. It has been concluded that caution should be exercised regarding eribulin use in elderly patients. In the present case, PR was achieved and maintained for 1 year with eribulin. Since the dose of eribulin was low, we could continue eribulin treatment for the long time without severe side effects. Moreover, this is the first case in which eribulin demonstrated an excellent antitumor effect for STS, suggesting that eribulin should be one of the most promising options for the treatment of STS. Further studies are needed to confirm the benefit of eribulin for patients with AS and to establish predictive markers for eribulin sensitivity.

\section{Acknowledgments}

Not applicable.

\section{Funding}

The current study was supported by a grant from the Japan Orthopaedics and Traumatology Research Foundation, Inc. (grant no. 372) and JSPS KAKENHI (grant no. JP19K18481).

\section{Availability of data and materials}

The datasets used and analyzed during the current study are available from the corresponding author on reasonable request.

\section{Authors' contributions}

YI and SN conceived the present study and wrote the manuscript. SN performed immunohistochemical analysis and pathological diagnosis of the patient. YI, TW, TT, HT, NN, and ST collected clinical data. YI and SN revised the manuscript. All authors read and approved the final manuscript.

\section{Ethics approval and consent to participate}

This study was approved by the ethics committee of our institution.

\section{Patient consent for publication}

Written informed consent was obtained from the patient for the publication of patient data and associated images.

\section{Competing interests}

The authors declare that they have no competing interests.

\section{References}

1. Young RJ, Brown NJ, Reed MW, Hughes D and Woll PJ: Angiosarcoma. Lancet Oncol 11: 983-991, 2010.

2. Stewart FW and Treves N: Lymphangiosarcoma in postmastectomy lymphedema; A report of six cases in elephantiasis chirurgica. Cancer 1: 64-81, 1948

3. McHaffie DR, Kozak KR, Warner TF, Cho CS, Heiner JP and Attia S: Stewart-treves syndrome of the lower extremity. J Clin Oncol 28: e351-e352, 2010.

4. Towle MJ, Salvato KA, Budrow J, Wels BF, Kuznetsov G, Aalfs KK, Welsh S, Zheng W, Seletsky BM, Palme MH, et al: In vitro and in vivo anticancer activities of synthetic macrocyclic ketone analogues of halichondrin B. Cancer Res 61: 1013-1021, 2001.

5. Jordan MA, Kamath K, Manna T, Okouneva T, Miller HP, Davis C, Littlefield BA and Wilson L: The primary antimitotic mechanism of action of the synthetic halichondrin E7389 is suppression of microtubule growth. Mol Cancer Ther 4: 1086-1095, 2005.

6. Okouneva T, Azarenko O, Wilson L, Littlefield BA and Jordan MA: Inhibition of centromere dynamics by eribulin (E7389) during mitotic metaphase. Mol Cancer Ther 7: 2003-2011, 2008.

7. Sharma A and Schwartz RA: Stewart-treves syndrome: Pathogenesis and management. J Am Acad Dermatol 67: 1342-1348, 2012.

8. Taswell HF, Soule EH and Coventry MB: Lymphangio-sarcoma arising in chronic lymphedematous extremities. Report of thirteen cases and review of literature. J Bone Joint Surg Am 44-A: 277-294, 1962.

9. Grobmyer SR, Daly JM, Glotzbach RE and Grobmyer AJ III: Role of surgery in the management of postmastectomy extremity angiosarcoma (stewart-treves syndrome). J Surg Oncol 73: 182-188, 2000.

10. Kerchner K, Fleischer A and Yosipovitch G: Lower extremity lymphedema update: Pathophysiology, diagnosis, and treatment guidelines. J Am Acad Dermatol 59: 324-331, 2008.

11. Judson I, Verweij J, Gelderblom H, Hartmann JT, Schöffski P, Blay JY, Kerst JM, Sufliarsky J, Whelan J, Hohenberger P, et al: Doxorubicin alone versus intensified doxorubicin plus ifosfamide for first-line treatment of advanced or metastatic soft-tissue sarcoma: A randomised controlled phase 3 trial. Lancet Oncol 15: 415-423, 2014. 
12. Fata F, O'Reilly E, Ilson D, Pfister D, Leffel D, Kelsen DP, Schwartz GK and Casper ES: Paclitaxel in the treatment of patients with angiosarcoma of the scalp or face. Cancer 86: 2034-2037, 1999.

13. Fury MG, Antonescu CR, Van Zee KJ, Brennan MF and Maki RG: A 14-year retrospective review of angiosarcoma: Clinical characteristics, prognostic factors, and treatment outcomes with surgery and chemotherapy. Cancer J 11: 241-247, 2005.

14. Skubitz KM and Haddad PA: Paclitaxel and pegylated-liposomal doxorubicin are both active in angiosarcoma. Cancer 104: 361-366, 2005.

15. Schlemmer M, Reichardt P, Verweij J, Hartmann JT, Judson I, Thyss A, Hogendoorn PC, Marreaud S, Van Glabbeke M and Blay JY: Paclitaxel in patients with advanced angiosarcomas of soft tissue: A retrospective study of the EORTC soft tissue and bone sarcoma group. Eur J Cancer 44: 2433-2436, 2008.

16. Penel N, Bui BN, Bay JO, Cupissol D, Ray-Coquard I, Piperno-Neumann S, Kerbrat P, Fournier C, Taieb S, Jimenez M, et al: Phase II trial of weekly paclitaxel for unresectable angiosarcoma: The ANGIOTAX study. J Clin Oncol 26: 5269-5274, 2008.

17. Nagano T, Yamada Y, Ikeda T, Kanki H, Kamo T and Nishigori C: Docetaxel: A therapeutic option in the treatment of cutaneous angiosarcoma: Report of 9 patients. Cancer 110: 648-651, 2007.

18. Stacchiotti S, Palassini E, Sanfilippo R, Vincenzi B, Arena MG, Bochicchio AM, De Rosa P, Nuzzo A, Turano S, Morosi C, et al: Gemcitabine in advanced angiosarcoma: A retrospective case series analysis from the Italian rare cancer network. Ann Oncol 23: 501-508, 2012.

19. Leu KM, Ostruszka LJ, Shewach D, Zalupski M, Sondak V, Biermann JS, Lee JS, Couwlier C, Palazzolo K and Baker LH: Laboratory and clinical evidence of synergistic cytotoxicity of sequential treatment with gemcitabine followed by docetaxel in the treatment of sarcoma. J Clin Oncol 22: 1706-1712, 2004.

20. Schoffski P, Chawla S, Maki RG, Italiano A, Gelderblom H, Choy E, Grignani G, Camargo V, Bauer S, Rha SY, et al: Eribulin versus dacarbazine in previously treated patients with advanced liposarcoma or leiomyosarcoma: A randomised, open-label, multicentre, phase 3 trial. Lancet 387: 1629-1637, 2016

21. Demetri GD, Schoffski P, Grignani G, Blay JY, Maki RG Van Tine BA, Alcindor T, Jones RL, D'Adamo DR, Guo M and Chawla S: Activity of eribulin in patients with advanced liposarcoma demonstrated in a subgroup analysis from a randomized phase III study of eribulin versus dacarbazine. J Clin Oncol 35 $3433-3439,2017$
22. Kawai A, Araki N, Naito Y, Ozaki T, Sugiura H, Yazawa Y, Morioka H, Matsumine A, Saito K, Asami S and Isu K: Phase 2 study of eribulin in patients with previously treated advanced or metastatic soft tissue sarcoma. Jpn J Clin Oncol 47: 137-144, 2017.

23. Wada N, Uchi H and Furue M: Case of angiosarcoma of the scalp successfully controled by eribulin. J Dermatol 45: 116-117, 2018.

24. Inagaki C, Shimoi T, Okuma H, Kitano A, Shimomura A, Noguchi E, Kodaira M, Yunokawa M, Yonemori K, Shimizu C, et al: A case of heavily pretreated metastatic cardiac angiosarcoma treated successfully using eribulin. Anticancer Drug 29: 97-101, 2018.

25. Kobayashi E, Naito Y, Asano N, Maejima A, Endo M, Takahashi S, Megumi Y and Kawai A: Interim results of a real-world observational study of eribulin in soft tissue sarcoma including rare subtypes. Jpn J Clin Oncol 49: 938-946, 2019.

26. Fujisawa Y, Fujimura T, Matsushita S, Yamamoto Y, Uchi H, Otsuka A, Funakoshi T, Miyagi T, Hata H, Gosho M, et al: The efficacy of eribulin mesylate for patients with cutaneous angiosarcoma previously treated with taxane: A multicentre prospective observational study. Br J Dermatol 2020 (Epub ahead of print).

(7) $\odot$ This work is licensed under a Creative Commons Attribution-NonCommercial-NoDerivatives 4.0 International (CC BY-NC-ND 4.0) License. 